\title{
Constraining the QCD phase diagram at finite temperature and density
}

\author{
Owe Philipsen* \\ Institut für Theoretische Physik, Goethe-Universität Frankfurt am Main \\ Max-von-Laue-Str. 1, 60438 Frankfurt am Main, Germany \\ E-mail: philipseneth.physik.uni-frankfurt.de
}

\begin{abstract}
Neither the chiral limit nor finite baryon density can be simulated directly in lattice QCD, which severely limits our understanding of the QCD phase diagram. In this review I collect results for the phase structure in an extended parameter space of QCD, with varying numbers of flavours, quark masses, colours, lattice spacings, imaginary and isospin chemical potentials. Such studies help in understanding the underlying symmetries and degrees of freedom, and are beginning to provide a consistent picture constraining the possibilities for the physical phase diagram.
\end{abstract}

37th International Symposium on Lattice Field Theory - Lattice2019

16-22 June 2019

Wuhan, China

${ }^{*}$ Speaker. 

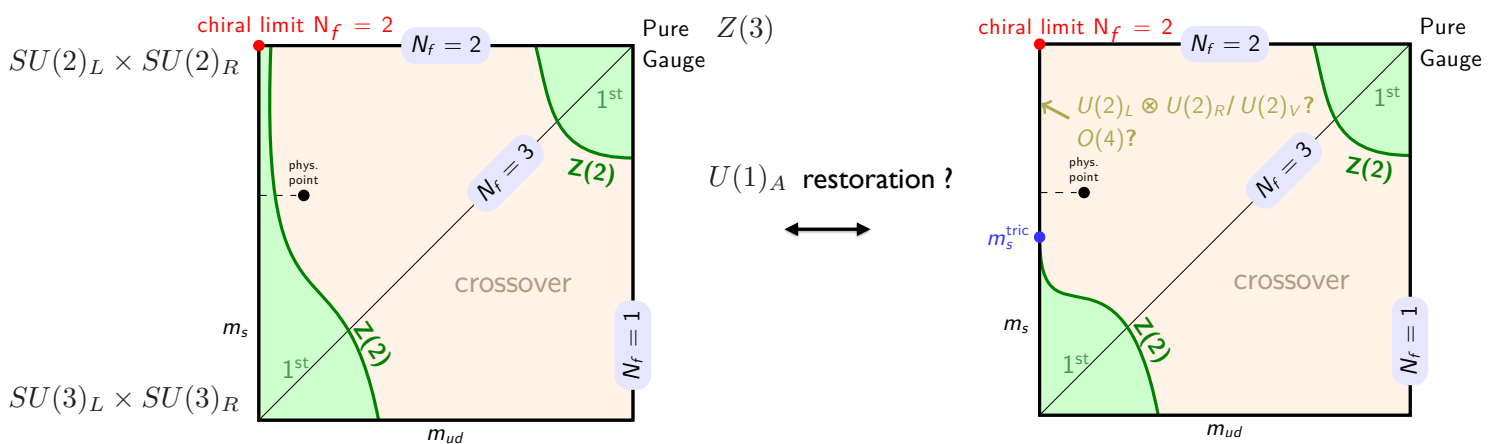

Figure 1: Possible scenarios for the order of the thermal QCD transition as a function of the quark masses.

\section{Introduction}

An outstanding open problem of QCD is the nature of its phase diagram as a function of temperature and baryon chemical potential. A change in dynamics from a hadron gas to a regime governed by different degrees of freedom, as in a quark gluon plasma, is expected to be caused by an effective restoration of chiral symmetry in a region with temperatures $T \lesssim 160 \mathrm{MeV}$ and baryon chemical potential $\mu_{B} \lesssim 1 \mathrm{GeV}$. Such low energy scales necessitate a non-perturbative firstprinciples approach like lattice QCD. Unfortunately, a severe sign problem prohibits simulations by importance sampling for non-vanishing chemical potential [1]. Despite tremendous efforts over several decades, no genuine solution to this problem is available.

In this contribution I will not cover the sign problem and the many attempts to alleviate it algorithmically. Instead I will report on a different strategy to learn about the phase diagram, which is to consider QCD thermodynamics in the parameter space $\left\{T, \mu_{B}, \mu_{I}, N_{f}, m_{q}^{f}, g^{2}, N_{c}\right\}$. By studying the phase structure in every parameter region, where some or another method works, an increasing number of constraints on the physical QCD phase diagram is obtained. As a by-product, such studies also provide theoretical insight about the interplay of the involved symmetries and degrees of freedom. I will begin with a discussion of the nature of the chiral phase transition in the chiral limit, before turning to proper finite density.

\section{The Columbia plot and its extended versions}

\subsection{The order of the thermal transition at zero density}

The nature of the thermal QCD transition with $N_{f}=2+1$ quark flavours as a function of the quark masses is summarised in the so-called Columbia plot, Fig. 1. In the quenched limit QCD reduces to $S U$ (3) Yang-Mills theory in the presence of static quarks and shows a first-order phase transition [2] associated with the spontaneous breaking of the $Z(3)$ center symmetry. Once quarks have a finite mass, the center symmetry is explicitly broken and the first-order phase transition weakens as the quarks get lighter, until it is lost at a $Z(2)$ second-order line of critical mass values.

In the opposite, chiral limit, the situation is more complicated, and for a long time expectations have been mostly guided by an analysis based on the epsilon expansion [3]. It predicts the chiral transition to be first-order for $N_{f} \geq 3$, whereas the case of $N_{f}=2$ is found to crucially depend on 

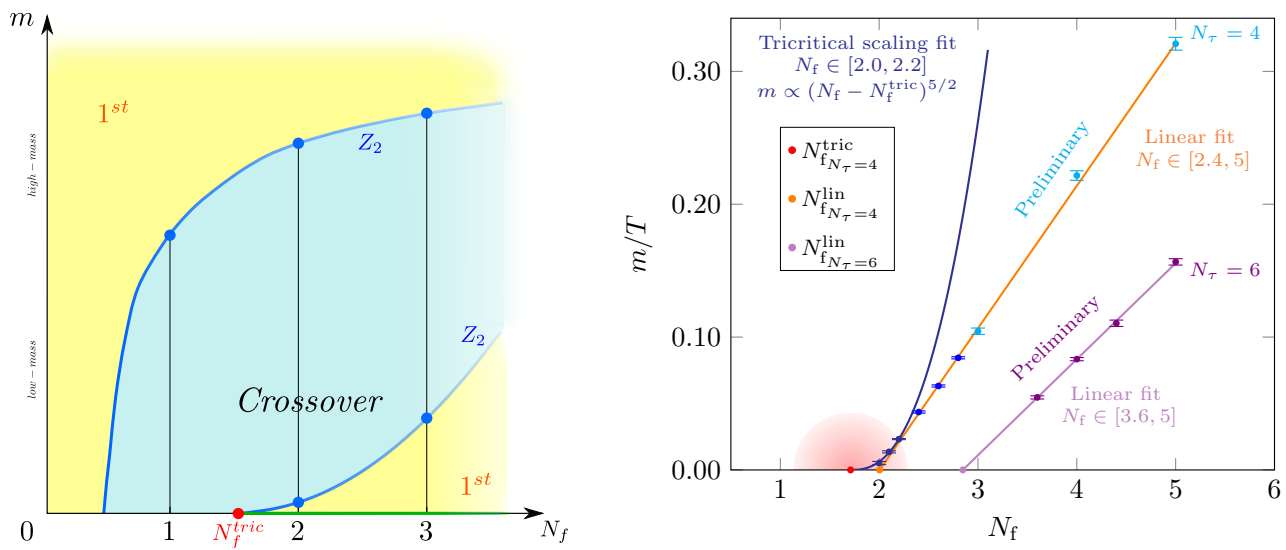

Figure 2: Left: Order of the thermal transition as function of $N_{f}$ and quark mass (schematic). Right: Second-order chiral critical line from unimproved staggered fermions [13].

the fate of the anomalous $U(1)_{A}$ symmetry: if the latter remains broken at $T_{c}$, the chiral transition should be second order in the $O(4)$-universality class, whereas its effective restoration would enlarge the chiral symmetry and push the transition to first-order. A later high order perturbative analysis of renormalisation group flow [4] instead finds a possible symmetry breaking pattern to be $U(2)_{L} \otimes U(2)_{R} \rightarrow U(2)_{V}$ in the case of a restored $U(1)_{A}$, which would amount to a second-order transition in a different universality class. For non-zero quark masses, chiral symmetry is explicitly broken and a first-order chiral transition weakens to disappear at a $Z(2)$ second-order critical boundary, while a second-order transition disappears immediately.

Computing these boundaries numerically is punishingly expensive. Locating a transition requires scans in temperature and mass, deciding its order and universality requires finite size scaling analyses with sufficiently large and different volumes. There is critical slowing down near a critical point as well as approaching the continuum, and the required quark masses are mostly smaller than physical. On coarse $N_{\tau}=4$ lattices, the first-order region is explicitly seen for $N_{f}=3$ unimproved staggered [5, 6] as well as $O(a)$-improved Wilson [7] fermions, the narrower $N_{f}=2$ region with unimproved staggered $[8,9]$ and unimproved Wilson [10] fermions. However, the location of the boundary line varies widely between these, indicating large cut-off effects. On the other hand, simulations with an improved staggered action (HISQ) do not see a first-order region on $N_{\tau}=6$ lattices even for $N_{f}=3$ [11]. The only point with a continuum extrapolation (besides the pure gauge limit) is the physical point, which has been identified to be in the crossover region [12].

\subsection{The chiral transition as a function of $N_{f}$}

In an alternative version of the Columbia plot, the chiral transition is considered as a function of the number of degenerate quark flavours and their mass. The first scenario from Fig. 1 then translates into Fig. 2 (left). The chiral symmetry and, with it, the strength of the transition increases with the number of flavours. One may now consider a partition function with the quark determinant raised to continuous, non-integer powers of $N_{f}$, in order to study the approach to the chiral limit [9]. For sufficiently large $N_{f}$, the chiral limit corresponds to a first-order, three-state coexistence line (with $\langle\bar{\psi} \psi\rangle=0, \pm$ const). The weakening of this transition with decreasing $N_{f}$ implies a tricritical point, in which it ends. The chiral critical line, which separates the first-order from the crossover region, enters the tricritical point with a known tricritical exponent. Of course, there is no physical 

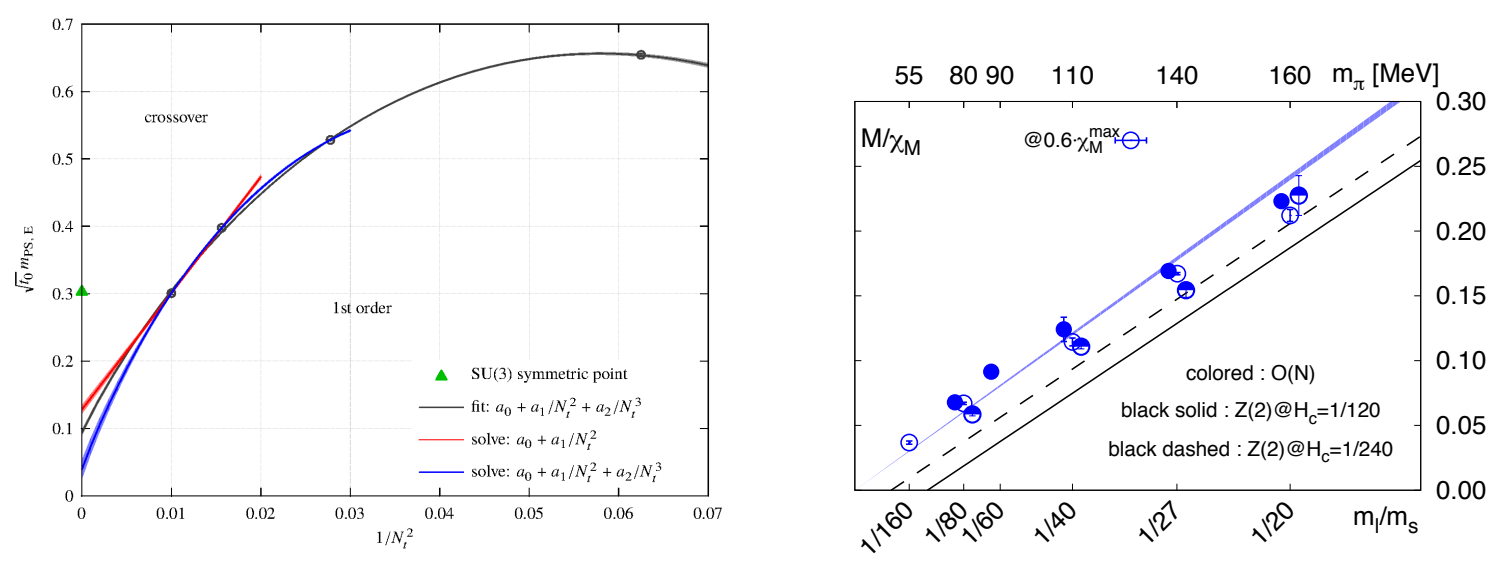

Figure 3: Left: Chiral critical point for $N_{f}=3 O(a)$ improved Wilson fermions [15]. Right: Magnetic equation of state approaching the chiral limit. Lines represent fits to $O(N)$ - (second-order scenario) or $Z(2)$ scaling with a finite critical quark mass (first-order scenario) [16].

meaning in this non-integer value of $N_{f}^{\text {tric }}$ other than to be smaller or larger than two, which puts the chiral transition for $N_{f}=2$ in the first- or second-order region, respectively.

A study on $N_{\tau}=4$ with unimproved staggered fermions [9] fully confirms these considerations, cf. Fig. 2 (right). For sufficiently small masses tricritical scaling is observed and can be used to extrapolate to the chiral limit. Unfortunately, the scaling region is small and little is gained over direct simulations at $N_{f}=2$. On $N_{\tau}=6$ lattices the scaling region is too low in quark mass to be simulated straightforwardly. However, beyond the scaling region the chiral critical line is observed to be linear over a large range of $N_{f}$-values. A linear extrapolation will then produce an upper bound for $N_{f}^{\text {tric }}$. In order to draw conclusions, one would need to know the size of the scaling region, where the chiral critical line is curved. In any case, a strong trend is seen for all $N_{f}$ : decreasing the lattice spacing dramatically shrinks the chiral critical quark mass bounding a first-order chiral transition. Similar observations up to $N_{\tau}=10$ are made for $N_{f}=4$ [14] and also for $N_{f}=3$ with $O(a)$-improved Wilson quarks [15] Fig. 3 (left).

While these calculations do not yet permit unambiguous continuum extrapolations, they provide a consistent picture across all discretisations: the chiral transition probed by lattice simulations weakens considerably as the continuum is approached. This makes a second-order scenario for the $N_{f}=2$ chiral limit more likely and raises the interesting question, whether also $N_{f}=3,4$ might possibly have second-order transitions, contrary to what was expected for a long time.

\subsection{From the physical point towards the chiral limit}

There is then no contradiction between unimproved and improved staggered actions, which do not see a first-order region so far. Recent investigations searching for the chiral phase transition with improved actions start at the $N_{f}=2+1$ physical point and then reduce the light quark masses. Thus either a $Z(2)$-critical point bounding the first-order region is approached, or a second-order transition in the chiral limit.

In $[16,17]$ the scaling behaviour was checked as the chiral limit is approached. Simulations were carried out using the HISQ action on lattices with $N_{\tau}=6,8,10$ and light quark masses down to $m_{\pi} \approx 55 \mathrm{MeV}$. The analysis uses a renormalisation group invariant combination of chiral con- 
densates as order parameter representing the magnetisation, and the light quark masses in units of the strange quark mass as symmetry breaking field,

$$
M=2\left(m_{s}\langle\bar{\psi} \psi\rangle_{l}-m_{l}\langle\bar{\psi} \psi\rangle_{s}\right) / f_{K}^{4}, \quad H=m_{l} / m_{s}
$$

Near a critical point the magnetic equation of state is then dominated by universal scaling functions

$$
M(t, h)=h^{1 / \delta} f_{G}(z)+\ldots, \quad \chi_{M}(t, h)=\frac{\partial M}{\partial H}=h_{0}^{-1} h^{1 / \delta-1} f_{\chi}(z)+\ldots
$$

with a scaling variable $z=t / h^{1 / \beta \delta}$ expressed in terms of the reduced temperature and external field, $t=t_{0}\left(T-T_{c}^{0}\right) / T_{c}^{0}, h=H / h_{0}$, which contain the unknown critical temperature in the chiral limit, $T_{c}^{0}$, and two non-universal parameters $t_{0}, h_{0}$.

Fig. 3 (left) shows the ratio $M / \chi_{M}$, whose approach to a critical point is sensitive to the scaling functions pertaining to the appropriate universality class. While it is difficult to distinguish between $O(4)$ and $Z(2)$, it is apparent that any finite critical quark mass bounding a first-order region has to be excessively small to be consistent with the observed behaviour. There is a similar scaling expression for the approach of the pseudo-critical crossover temperature of some observable $X$ to the critical temperature in the chiral limit,

$$
T_{X}(H)=T_{c}^{0}+\frac{z_{X}}{z_{0}} T_{c}^{0} H^{1 / \beta \delta}, \quad T_{c}^{0}=132_{-6}^{+3} \mathrm{MeV} .
$$

In [17] the variation with the possible critical exponents is observed to be very small, so that an extrapolation makes sense even without definite knowledge of the eventual universality class. Results were checked to be stable when the continuum extrapolation is done before the chiral extrapolation, leading to the critical temperature as a first result for the chiral limit. Note that its value is $\sim 25 \mathrm{MeV}$ lower than the pseudo-critical temperature at the physical point, which should be important for phenomenological descriptions of chiral symmetry breaking.

Similar conclusions are drawn in an exploratory study attempting to approach the chiral limit more economically by reweighting in the light quark mass [18], with a weight factor

$$
W=\frac{\operatorname{det}(D)}{\operatorname{det}\left(D+m_{u d}\right)}=\exp \left[-\frac{V}{T} m_{u d} \bar{\psi} \psi\left(m_{u d}\right)+O\left(m_{u d}^{4}\right)\right] .
$$

The Banks-Casher relation is used to relate the chiral condensate to the spectral density of the Dirac operator, which is then reweighted to zero mass. Note, that this involves an infinite volume extrapolation by polynomial fits, whose systematics still needs studying. Nevertheless, the finite size scaling of the chiral condensate obtained in this approach clearly prefers a second-order scenario.

\subsection{The $U(1)_{A}$ anomaly}

As pointed out in section 2.1, the fate of the anomalous $U(1)_{A}$ around the thermal transition is expected to play a significant role in determining the order of the chiral phase transition. Simulations of a model realising the QCD chiral symmetry with a tuneable strength of the $U(1)_{A}$ anomaly indeed demonstrate that a first-order transition occurs for restored symmetry, which changes to an $O(4)$-transition above some critical strength of symmetry breaking [19]. Unfortunately, this 

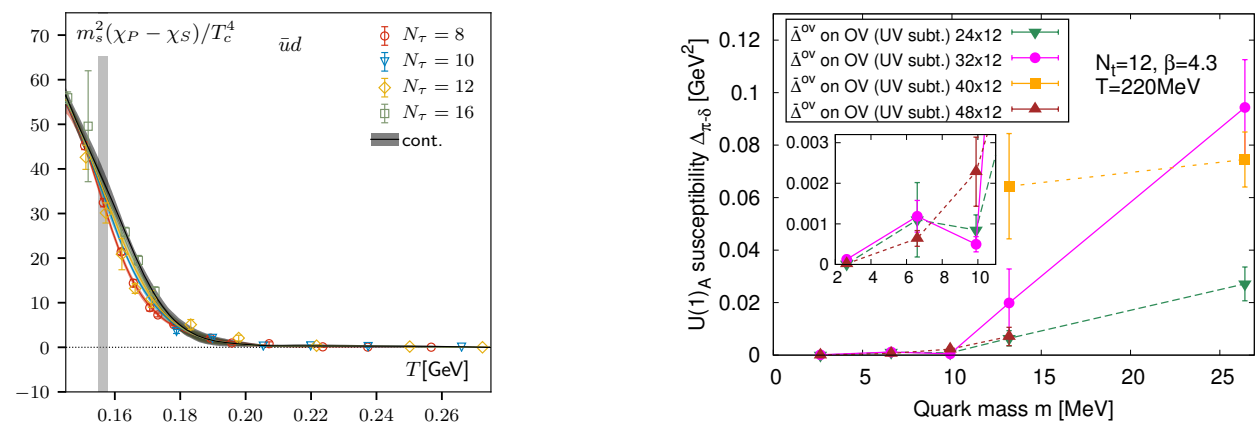

Figure 4: Splitting between scalar and pseudo-scalar susceptibilities. Left: Overlap operator evaluated on HISQ ensembles at the physical point [23]. Right: DW fermions reweighted to overlap [24].

"critical strength" of symmetry breaking is nothing universal that could be easily mapped to QCD, which thus has to be investigated directly.

Several attempts over the last years used using different discretisation schemes, from which apparently contradicting conclusions were drawn. Studies with chiral domain wall fermions on $N_{\tau}=8$ with $200 \mathrm{MeV}$ pions [20] find evidence of a broken $U(1)_{A}$ at the pseudo-critical temperature, and the same result is reported when evaluating the overlap operator on $N_{\tau}=8 \mathrm{HISQ}$ ensembles with $160 \mathrm{MeV}$ pions [21]. On the other hand, the screening mass spectrum evaluated at the pseudo-critical temperature with $O(a)$-improved Wilson fermions on fine $N_{\tau}=16$ lattices suggests a significant reduction of the anomaly in the chiral limit [22], and Möbius domain wall fermions reweighted to overlap on $N_{\tau}=8,10$ show full symmetry restoration at $T \sim 220 \mathrm{MeV}$. Updates on these results, using the difference of scalar and pseudo-scalar susceptibilities as a measure for the anomaly,

$$
\Delta_{\pi-\delta}=\chi_{\pi}-\chi_{\delta}=\int d^{4} x\left\langle\pi^{a}(x) \pi^{a}(0)-\delta^{a}(x)-\delta^{a}(0)\right\rangle,
$$

are shown in Fig. 4. On the left the overlap Dirac operator is evaluated on HISQ ensembles with physical quark masses, including an extrapolation to the continuum limit [23]. The full restoration of the symmetry happens above the chiral crossover temperature, but this is not yet the chiral limit. Fig. 4 (right) shows the same quantity as a function quark mass, with domain wall fermions reweighted to overlap on $N_{\tau}=12$ at $T=220 \mathrm{MeV}$ [24]. The splitting is observed to vanish in the chiral limit, but in this case the temperature is above the transition. The qualitative features of both calculations are fully consistent and no contradiction is apparent yet. It would be most valuable to compare both approaches for a set of identical parameter values. For future investigations it might also be useful to check further relations between chiral and $U(1)_{A}$ restoration, provided by certain Ward identities, which give information on the scaling with temperature as a criterion for exact symmetry restoration [25].

\subsection{QCD with imaginary chemical potential}

While it is unphysical, imaginary chemical potential for quark number, $\mu=i \mu_{i}, \mu_{i} \in \mathbb{R}$, does not induce a sign problem and thus can be simulated without difficulty. This has been used to extract several aspects of the low density phase diagram at real $\mu$ by analytic continuation [26, 27]. Two exact symmetries facilitate such studies. Because of $C P$-invariance, the QCD partition function is 

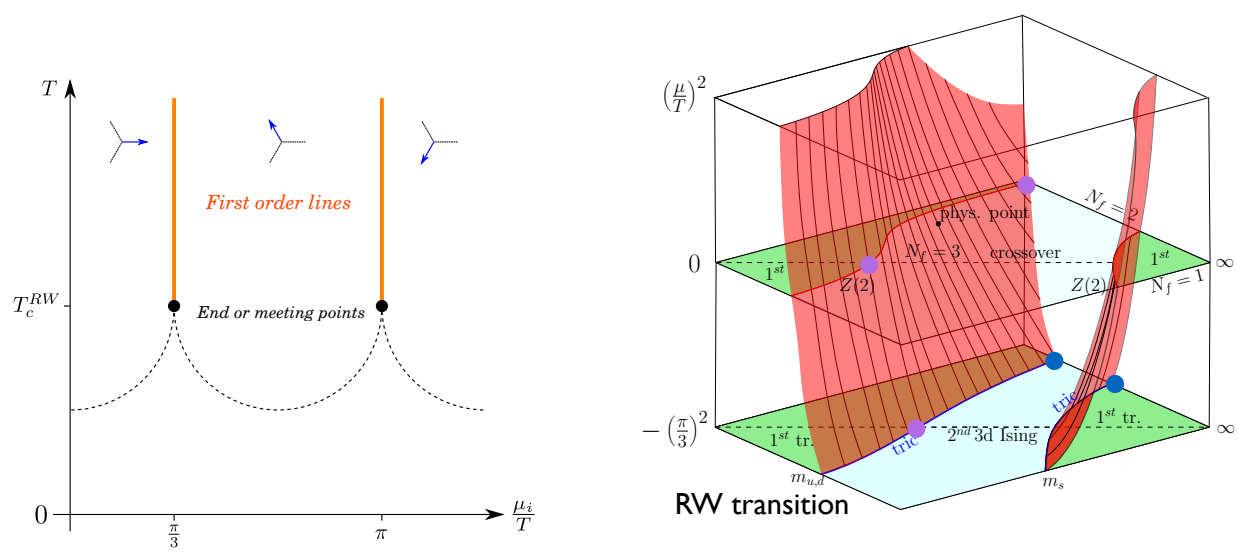

Figure 5: Left: The QCD phase diagram at imaginary chemical potential. Vertical lines mark first-order transitions between different center sectors, the dotted lines are the analytic continuation of the transitions at real $\mu$, whose nature depends on the quark masses. Right: Columbia plot with chemical potential. The bottom plane corresponds to the first center-transition.

even in chemical potential $Z(\mu)=Z(-\mu)$. Furthermore, for arbitrary fermion masses it is periodic in imaginary chemical potential because of the global Roberge-Weiss (or center) symmetry [28],

$$
Z\left(T, i \frac{\mu_{i}}{T}\right)=Z\left(T, i \frac{\mu_{i}}{T}+i \frac{2 \pi n}{N_{c}}\right) .
$$

This leads to transitions cycling through the $N_{c}$ center sectors, which are distinguishable by the phase of the Polyakov loop but have equal thermodynamic functions. The transitions are first order for high temperatures and crossover for low temperatures, see Fig. 5 (left). In the $T$-direction there is the analytic continuation of the QCD thermal transition, whose order depends on $N_{f}$ and the quark masses. For first-order chiral and deconfinement transitions (small and large quark masses), the transition lines meet up in a triple-point, while for thermal crossover the RW-transition ends in a critical endpoint with $3 \mathrm{~d}$ Ising universality. The boundary between these scenarios is marked by a tricritical point. These structures have been established explicitly for unimproved staggered $[29,30]$ as well as unimproved Wilson [31] fermions.

This translates into a $3 \mathrm{~d}$ extension of the Columbia plot, as in Fig. 5 (right). Regions of chiral and deconfinement phase transitions are now separated by critical surfaces from the crossover region. The curvature of the chiral critical surface has been shown to be negative both on the $N_{f}=3$ diagonal, as well as near the physical point [32] on $N_{\tau}=4$ lattices. Thus the chiral transition strengthens with imaginary and weakens with real chemical potential. This is opposite to a scenario with a chiral critical point close to the temperature axis, which would require the chiral transition at the physical point to stregthen with real $\mu$. Unfortunately, because of the receding first-order region, these calculations could not yet be repeated on finer lattices.

Investigations on finer lattices reveal the same trend as seen at $\mu=0$, namely the chiral tricritical line moving towards smaller quark masses, both for unimproved staggered [33] and Wilson [34] quarks, Fig. 6 (left). For stout-smeared staggered [35] and HISQ [36] actions on $N_{\tau}=4$, even the larger first-order region in the RW-plane cannot be detected when starting from the physical point and reducing the pion masses down to $m_{\pi} \approx 50 \mathrm{MeV}$, as Fig. 6 (right) demonstrates with second-order scaling. 

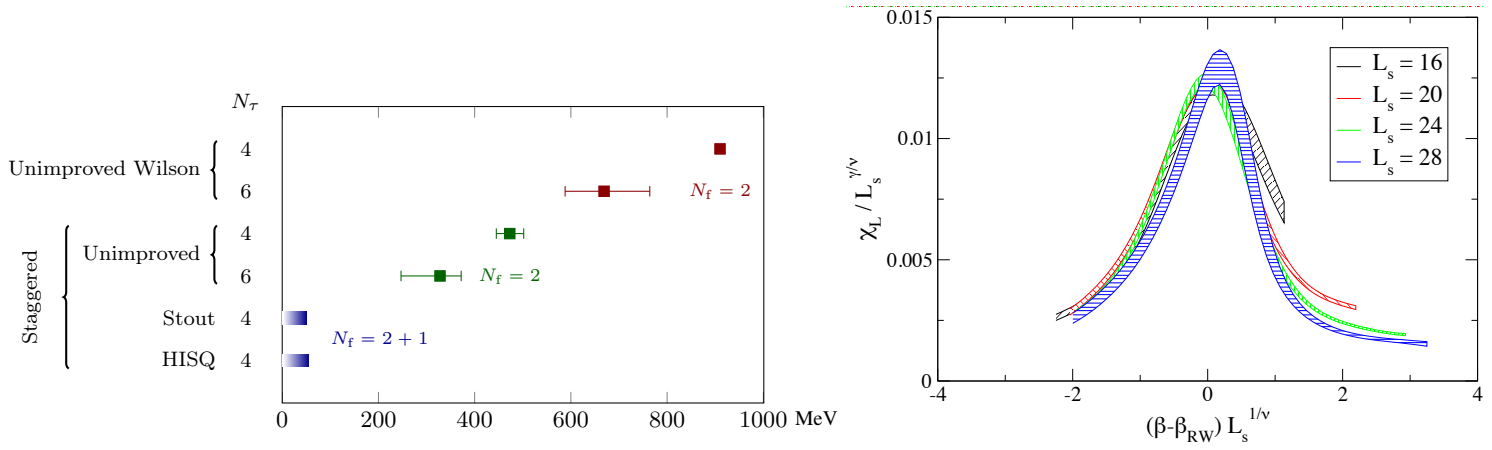

Figure 6: Left: Tricritical pion mass values delimiting the first-order chiral region in the RW-plane [33]. Right: Finite size scaling with $3 \mathrm{~d} Z(2)$ exponents for stout-smeared staggered fermions with $m_{\pi} \approx 50 \mathrm{MeV}$ and $\left(m_{u d} / m_{s}\right)_{\text {phys }}$ on $N_{\tau}=4$ in the RW-plane [35].

Together with the $\mu=0$ results, this means that the entire chiral critical surface is shifting drastically towards the chiral limit as the lattice spacing is decreased, and it is an open question whether any first-order transition remains in the continuum limit. At the same time, this implies a softening of the crossover at the physical point, with so far no indication of a chiral critical structure at real chemical potential.

\section{QCD at the physical point}

\subsection{Emerging chiral spin symmetry}

Besides the location and nature of phase transitions, studies of phase diagrams are also concerned with an identification of the dominant dynamical degrees of freedom in each regime, which are expected to reflect the underlying symmetries. In QCD, in particular, the hadronic regime is usually associated with broken chiral symmetry, while the quark gluon plasma represents a symmetry restored state. In this context new investigations point to an interesting intermediate temperature regime with an emerging $S U(4)$ symmetry, which was first proposed in [37].

Consider a $S U(2)_{C S}$ chiral spin transformation of quark fields defined by

$$
\psi(x) \rightarrow \exp (i \vec{\Sigma} \cdot \vec{\varepsilon}), \quad \Sigma_{k}=\left\{\gamma_{k},-i \gamma_{5} \gamma_{k}, \gamma_{5}\right\} .
$$

The QCD Lagrangian is not invariant under such transformations. However, when there is a thermodynamic medium implying a preferred Lorentz frame, one finds the colour-electric part of the quark-gluon interaction as well as a chemical potential term for fermion number to be invariant, while kinetic terms and colour-magnetic interactions are not. Combining chiral spin symmetry with isospin, $S U(2)_{C S} \times S U(2)$, it can be embedded in a $S U(4)$ symmtery that fully contains the usual chiral symmetry of the Lagrangian, $S U(4) \supset S U(2)_{L} \times S U(2)_{R} \times U(1)_{A}$.

The realisation of these symmetries has recently been tested with spatial [38] and temporal [39] correlation functions. Fig. 7 shows some examples of spatial correlators,

$$
C_{\Gamma}\left(n_{z}\right)=\sum_{n_{x}, n_{y}, n_{\tau}}\left\langle O_{\Gamma}\left(n_{x}, n_{y}, n_{z}, n_{\tau}\right) O_{\Gamma}(\mathbf{0}, 0)\right\rangle,
$$

with quantum numbers specified by $\Gamma$, evaluated on JLQCD configurations with $N_{f}=2$ domain wall fermions with physical light quark masses from $N_{\tau}=4,6,8,12$ lattices. At $T=220 \mathrm{MeV}$, 

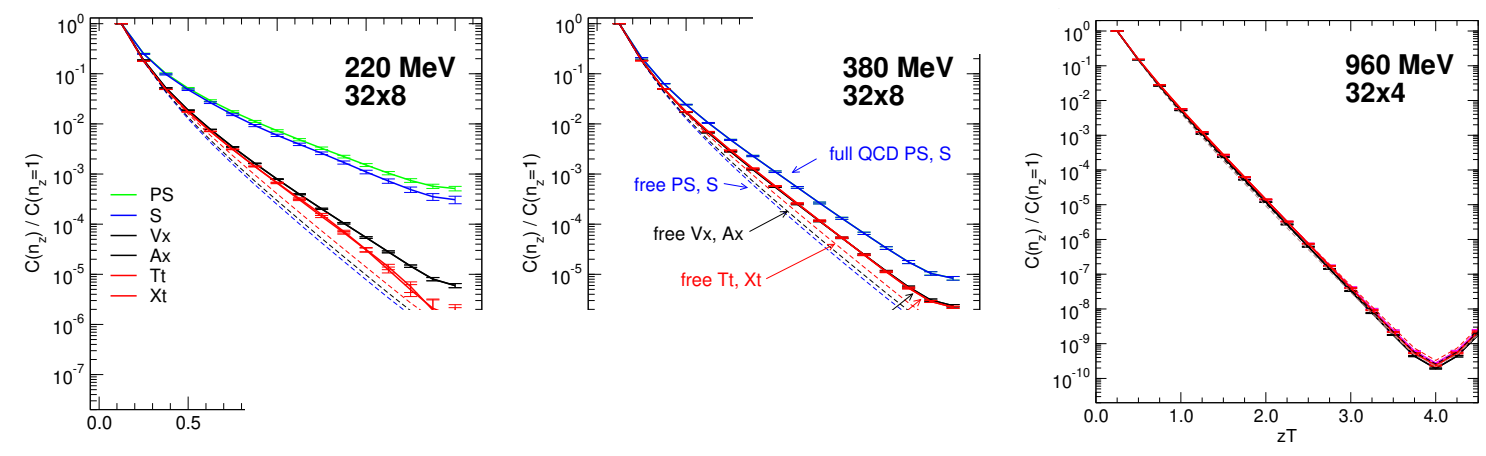

Figure 7: Spatial correlation functions of the $E_{1}, E_{2}$ multiplets of the $S U(4)$ chiral spin symmetry, as a function of temperature and in comparison to free quark correlators (dashed lines) [38].

i.e. above the chiral crossover, a near-degeneracy pattern of different quantum number channels is observed, which is consistent with the multiplets of the $S U(4)$-symmetry worked out in [38]. As the temperature is increased to $380 \mathrm{MeV}$, these multiplets move closer to each other, while in both cases they differ clearly from the leading-order perturbative pattern expected for free quarks. Finally, as the temperature approaches $\sim 1 \mathrm{GeV}$, the different multiplets fall on top of each other and approach those of free quark correlators, signalling restoration of the full chiral symmetry.

This has implications for the active degrees of freedom in the temperature range just above the chiral crossover. The nearly intact multiplet structure implies suppression of colour-magnetic interactions, and the authors interpret the active degrees of freedom as chiral quarks bound to colour singlets by colour-electric strings. For this reason they term this regime, which has more resemblance to a hadron gas than to a plasma, a "stringy fluid".

This finding based on an emergent chiral symmetry is in fact consistent with earlier studies distinguishing between colour-electric and -magnetic degrees of freedom by the discrete transformation of Euclidan time reversal (or $C T$ in Minkowski time) [40]. The lowest screening masses in the framework of dimensionally reduced QCD, valid for temperatures above the crossover, and thus the dominant degrees of freedom, correspond to colour-electric operators, with colour magnetic ones contributing to excited states only [41]. This is contrary to the perturbative ordering of electric and magnetic scales, $\sim g T, g^{2} T$, respectively, which is only realised at much higher temperatures. Finally, the picture of a hadron-like stringy fluid is also consistent with sequential melting scneario of heavy quarkonia at $T>T_{p c}$.

\subsection{The crossover at small baryon densities}

There are three methods that have been used so far to extract information about the phase structure at the physical point for small baryon density. All of them introduce some approximation which can be controlled as long as $\mu / T \lesssim 1$ : i) reweighting [42], ii) Taylor expansion in $\mu / T$ [43] and iii) anlaytic continuation from imaginary chemical potential $[26,27]$. When the QCD pressure is expressed as a series in baryon chemical potential,

$$
\frac{p\left(T, \mu_{B}\right)}{T^{4}}=\frac{p(T, 0)}{T^{4}}+\sum_{n=1}^{\infty} \frac{1}{2 n !} \chi_{2 n}^{B}(T)\left(\frac{\mu_{B}}{T}\right)^{2 n}, \quad \chi_{2 n}^{B}(T)=\left.\frac{\partial^{2 n}\left(\frac{p}{T^{4}}\right)}{\partial\left(\frac{\mu_{B}}{T}\right)^{2 n}}\right|_{\mu_{B}=0},
$$

the Taylor coefficients are the baryon number fluctuations evaluated at zero density, which can also be computed by fitting to untruncated results at imaginary $\mu_{B}$, thus permitting full control of the 

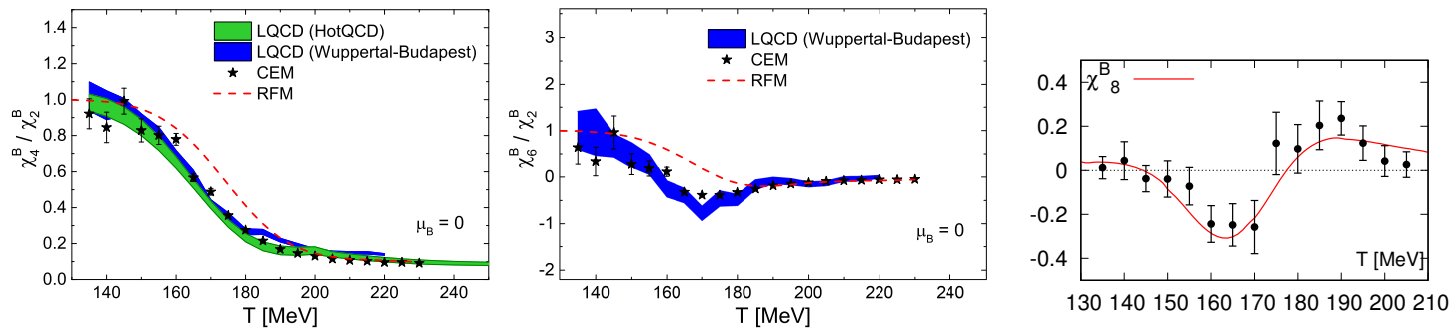

Figure 8: Baryon number fluctuations $\chi_{4}^{B}, \chi_{6}^{B}$ from the lattice in comparison with CEM, RFM models [44] (left, middle), and $\chi_{8}^{B}$ fitted with a polynomial model without criticality [45] (right).

systematics between ii) and iii). They are presently known up to $2 n=8$ on $N_{\tau}=16$ lattices, Fig. 8 , and in principle also observable experimentally. For a review of the equation of state relating to heavy ion phenomenology, see [46]. Note also, that this regime appears now accessible by complex Langevin simulations without series expansion, albeit not yet for physical quark masses [47]. From the susceptibility of an appropriately normalised chiral condensate follows the pseudocritical temperature, similarly as a power series. The latest continuum extrapolated results are

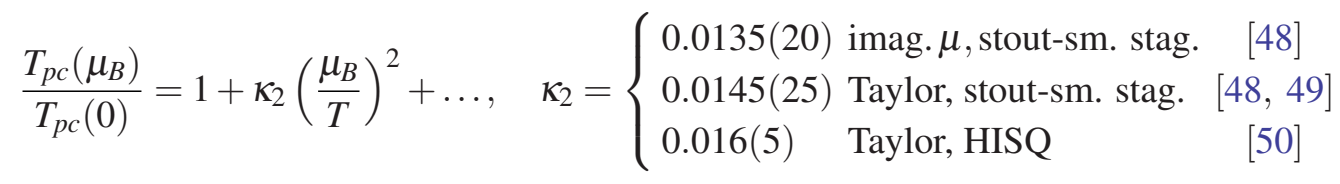

with $T_{p c}(0)=156.5(1.5) \mathrm{MeV}$ [50], and the sub-leading term insignificant to current accuracy.

\subsection{The radius of convergence}

If a function with a given domain of analyticity in its complex argument is expanded in a power series, the radius of convergence specifies the distance between the expansion point and the nearest singularity. This implies that the location $\left(T_{c}, \mu_{B}^{c}\right)$ of a non-analytic QCD phase transition constitutes an upper bound on the radius of convergence of the pressure series (3.3). It has been argued in the literature that this can be turned around in order to search for a critical point: if a finite radius of convergence can be extracted from the pressure series for real parameter values, it should signal a phase transition. The standard estimator used in the literature is the ratio test of consecutive coefficients, whose extrapolation to infinite order yields the radius of convergence,

$$
r=\lim _{n \rightarrow \infty} r_{2 n}, \quad r_{2 n}=\left|\frac{2 n(2 n-1) \chi_{2 n}^{B}}{\chi_{2 n+2}^{B}}\right| .
$$

In practice, only the first few coefficients are available. Nevertheless, several constraints on a critical endpoint have been based on $r_{2 n}$ and published, a recent compilation can be found in [51].

However, it has recently become clear in model studies that the ratio estimator is inappropriate for the case at hand. As an example, consider the fugacity expansion of baryon number density. At imaginary chemical potential, this is just a Fourier series whose coefficients can be computed on the lattice without sign problem,

$$
\left.\frac{n_{B}}{T^{3}}\right|_{\mu_{B}=i \theta_{B} T=}=i \sum_{k} b_{k}(T) \sin \left(k \mu_{B} / T\right), \quad b_{k}(T)=\frac{2}{\pi} \int_{0}^{\pi} d \theta_{B} \operatorname{Im}\left(\frac{n_{B}\left(T, i \theta_{B} T\right)}{T^{3}} \sin \left(k \theta_{B}\right) .\right.
$$



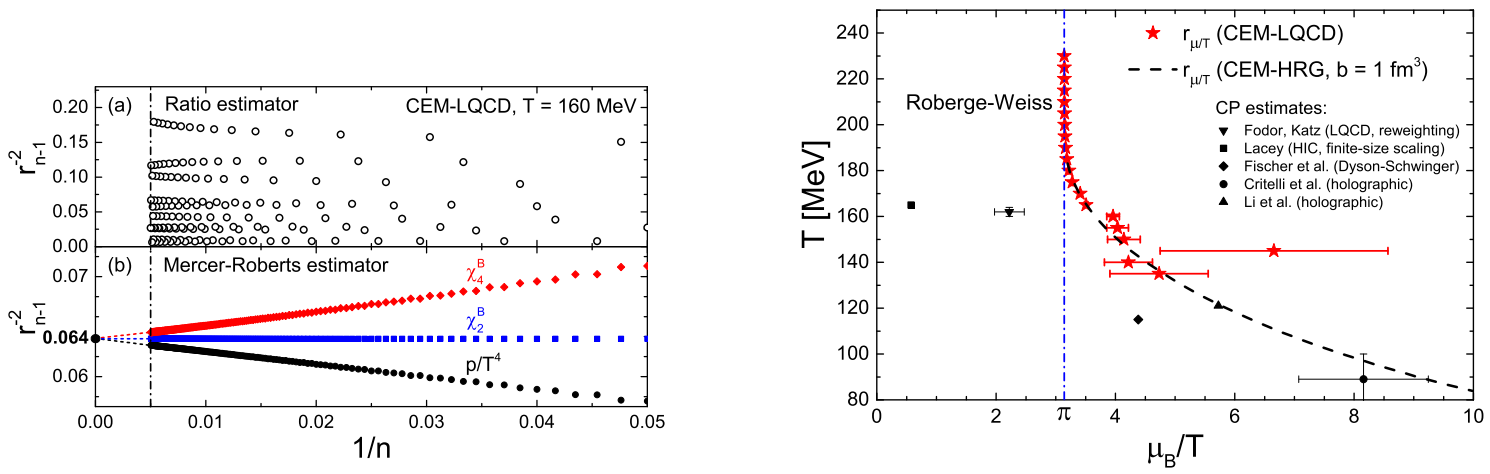

Figure 9: Left: Comparison of estimators for the radius of convergence in a Cluster Expansion Model. Right: The CEM radius of convergence as a function of $T$ predicts the RW-transition at imaginary $\mu_{B}$ [52].

In [52] a cluster expansion model (CEM) was proposed, which takes the first two coefficients as input from a lattice calculation [53], and expresses all higher coefficients in terms of these,

$$
b_{k}(T)=\alpha_{k}^{S B} \frac{\left[b_{2}(T)\right]^{k-2}}{\left[b_{1}(T)\right]^{k-1}}, \quad k=3,4, \ldots
$$

The $\alpha_{k}^{S B}$ are $T$-independent and fixed to reproduce the Stefan-Boltzmann limit. Hence, this includes two-body interactions only, corresponding to NLO in a virial expansion, which should be valid at sufficiently high temperatures and low densities. Of course, modelling higher coefficients in terms of the lower ones is not unique, an alternative is provided by the rational function model (RMF) [54]. The model now predicts the coefficients $b_{k \geq 3}$ or any of the baryon number susceptibilities, a closed expression to all orders as a polylogarithm is also available [44]. All existing lattice data are reproduced surprisingly accurately, as the examples in Fig. 8 show.

With all coefficients of the fugacity expansion available, one can study the radius of convergence of CEM, Fig. 9. The ratio estimator fails to converge, because of the irregular signs of higher order coefficients (it works for equal or alternating signs). On the other hand, the Mercer-Roberts estimator, which uses three consecutive coefficients of a series [55],

$$
r_{n}=\left|\frac{c_{n+1} c_{n-1}-c_{n}^{2}}{c_{n+2} c_{n}-c_{n+1}^{2}}\right|^{1 / 4},
$$

converges and extrapolates to a unique radius of convergence, independent of the observable used. Fig. 9 (right) shows the result for various temperatures, which intriguingly predicts the RobergeWeiss transition in the direction of imaginary chemical potential. Conversely, this implies that the CEM has no phase transition for real $\mu_{B} \leq \pi T$. Of course, this is a model and does not exclude a QCD critical point in this range. (For an application of the RFM to a chiral model which has a phase transition, see [54].) However, the analysis does imply that there is no sign of criticality in the presently available lattice data at zero or imaginary chemical potential (see also Fig. 8 (right)).

A more general study investigates how the singularity structure of QCD is reflected in LeeYang zeroes and the radius of convergence [56]. It concludes that the ratio test fails in any finite volume and also advocates the Mercer-Roberts as well as the Cauchy-Hadamard estimators. Improved versions are constructed for these estimators with enlarged sensitivity to the closest singu- 

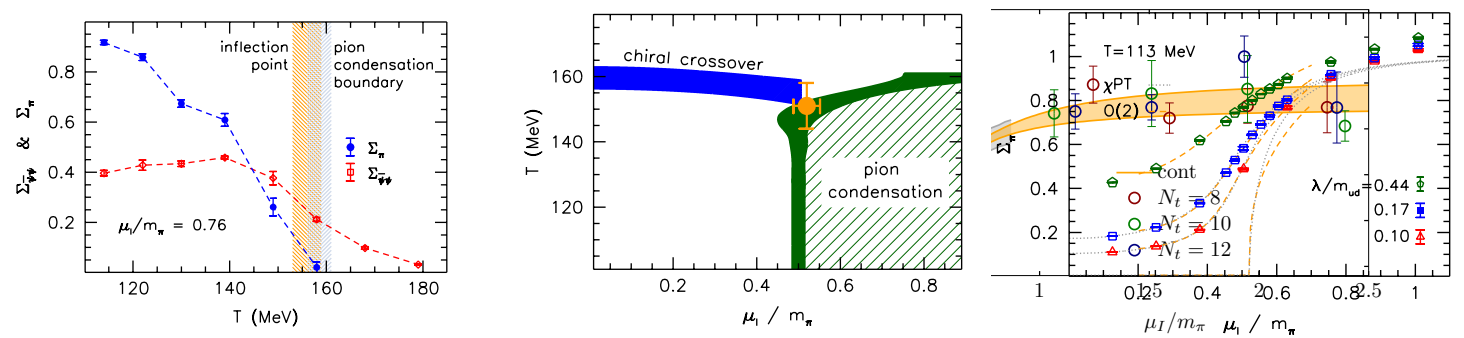

Figure 10: Left: Chiral and isospin condensated as a function of $T$. Middle: Phase diagram at finite isospin chemical potential. Right: Scaling of the pion condensate as a signal for a second-order transition [63].

larity, which are able to pick up the known phase transition of $N_{f}=4$ staggered QCD on a $N_{\tau}=4$ lattice with less than ten coefficients.

Having to go term by term in an expansion can be avoided, if the Lee-Yang zero closest to the origin can be determined directly. Using reweighting, this was the strategy employed in the first prediction of a critical point on $N_{\tau}=4$ lattices using unimproved rooted staggered fermions [57]. However, a new investigation points out that, for this discretisation, the closest Lee-Yang zero is caused by a spectral gap between the unrooted tastes rather than by a phase transition [58]. A new definition of the rooted staggered determinant at finite $\mu$ is suggested, which avoids these artificial non-analyticies. Application to the stout-smeared action on $N_{\tau}=4$, again using reweighting, shows the closest singularity to be off the real axis, pushing a possible phase transition beyond $\mu_{B} \gtrsim 2 T$.

\subsection{Finite isospin density}

It was noted long ago that QCD at finite isospin density does not have a sign problem and can be simulated directly [59]. Finite isospin is also physically relevant for neutron stars or the early universe with a lepton asymmetry. Consider the (degenerate) light quark action in the form

$$
S=\bar{\psi}\left(\gamma_{\mu}\left(\partial_{\mu}+i A_{\mu}\right)+m_{u d}+\mu_{I} \gamma_{4} \tau_{3}+i \lambda \gamma_{5} \tau_{2}\right) \psi, \quad \mu_{I}=\left(\mu_{u}-\mu_{d}\right) / 2
$$

A chemical potential for isospsin is realised by having chemical potentials of opposite sign for the $u$ - and $d$-quarks. For $\mu_{I}=0$, the action is invariant under a $S U(2)_{V} \times U(1)_{V}$ associated with isospin and baryon number, which gets broken to $U(1)_{\tau_{3}} \times U(1)_{V}$ by $\mu_{I} \neq 0$. In this case there is a further spontaneous symmety breaking leaving only the baryon $U(1)_{V}$, signalled by a nonvanishing expectation value for the charged pions,

$$
\left\langle\pi^{ \pm}\right\rangle=\left\langle\bar{\psi} \gamma_{5} \tau_{1,2} \psi\right\rangle
$$

which then correspond to Goldstone modes. The $\lambda$-term in the action introduces an explicit breaking of the remaining symmetry and is necessary for simulation purposes only, in order to pick one of the degenerate vacua. For physical results, simulations have to be extrapolated to $\lambda \rightarrow 0$, a task quite similar to approaching the chiral limit at $\mu=0$, and hence difficult.

After several exploratory studies (e.g. [60, 61, 62]) new calculations are for $N_{f}=2+1$ quarks with physical masses using a stout-smeared staggered action, on lattices with $N_{\tau}=6,8,10,12$, followed by a continuum extrapolation. The $\lambda \rightarrow 0$ extrapolation is done with a similar reweighting technique applied to a singular value representation of the pion condensate, as for the chiral 

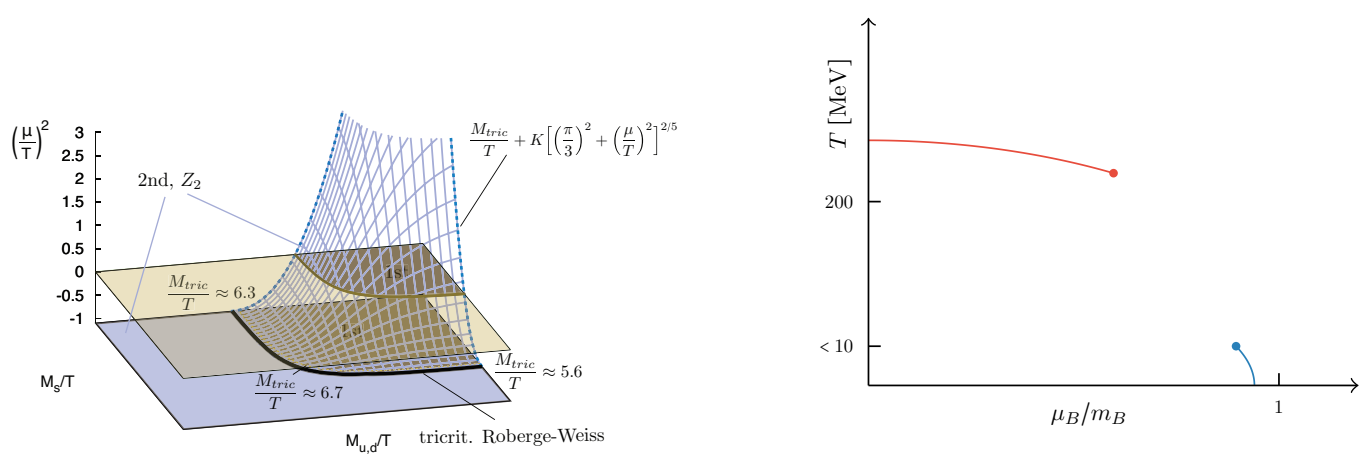

Figure 11: Left: Heavy mass corner of the Columbia plot, computed in an effective theory [68]. Right: Schematic phase diagram for QCD with heavy quarks.

condensate mentioned in section 2.3 [18]. The order parameters used to characterise the phase structure are the chiral and pion condensates,

$$
\Sigma_{\pi}=\frac{m_{u d}}{m_{\pi}^{2} f_{\pi}^{2}}\left\langle\pi^{ \pm}\right\rangle, \quad \Sigma_{\bar{\psi} \psi}=\frac{m_{u d}}{m_{\pi}^{2} f_{\pi}^{2}}\left(\langle\bar{\psi} \psi\rangle_{T, \mu_{I}}-\langle\bar{\psi} \psi\rangle_{0,0}\right)+1
$$

which are shown as a function of $T$ for a specific choice of $\mu_{I}$ in Fig. 10 (left). Varying both thermodynamic parameters, the phase boundaries have been mapped out leading to the phase diagram in Fig. 10 (middle). For the transition in the $\mu_{I}$-direction, the extrapolation $\lambda \rightarrow 0$ is consistent with $O(2)$ scaling of the pion condensate, identifying the transition to be second-order. Prospects to reweight in $\mu_{B}$ using finite isospin as a point of departure are discussed in [64].

\section{Larger baryon density via effective lattice theories}

For the cold and dense regime, $\mu_{B} / T \gg 1$, where the sign problem is strongest, no genuine methods are available. Nevertheless, some progress towards at least qualitative physics has been made over the last few years by means of effective theories. The general idea is to split the problem in two parts: first derive an effective theory by expansion methods in some small parameter. By this step some degrees of freedom have already been integrated over, such that the sign problem for the resulting effective theory is milder than the original one. In a second step the effective theory is solved by flux representations simulated by a worm algorithm, complex Langevin simulations or analytic series expansion methods.

Two types of effective degrees of freedom arise naturally, depending on the integration order,

$$
Z=\int D U D \bar{\psi} D \psi e^{-S_{Q C D}[U, \bar{\psi}, \psi]}=\int D U_{0} e^{-S_{e f f}\left[U_{0}\right]}=\int D \bar{\psi} D \psi e^{-S_{e f f}[\bar{\psi}, \psi]} .
$$

In the first case, fermions are integrated over as well as all spatial link variables, leaving a theory of temporal links only, which on a periodic lattice can be expressed by Polyakov loops. In the second case, all gauge links are integrated, leaving a fermionic effective theory in terms of mesons and baryons, because of gauge invariance. Note that both representations are perfectly equivalent to QCD. Because of the truncations involved in doing the integrations analytically, this equivalence is reduced to specific parameter regions, where the approximations hold. 

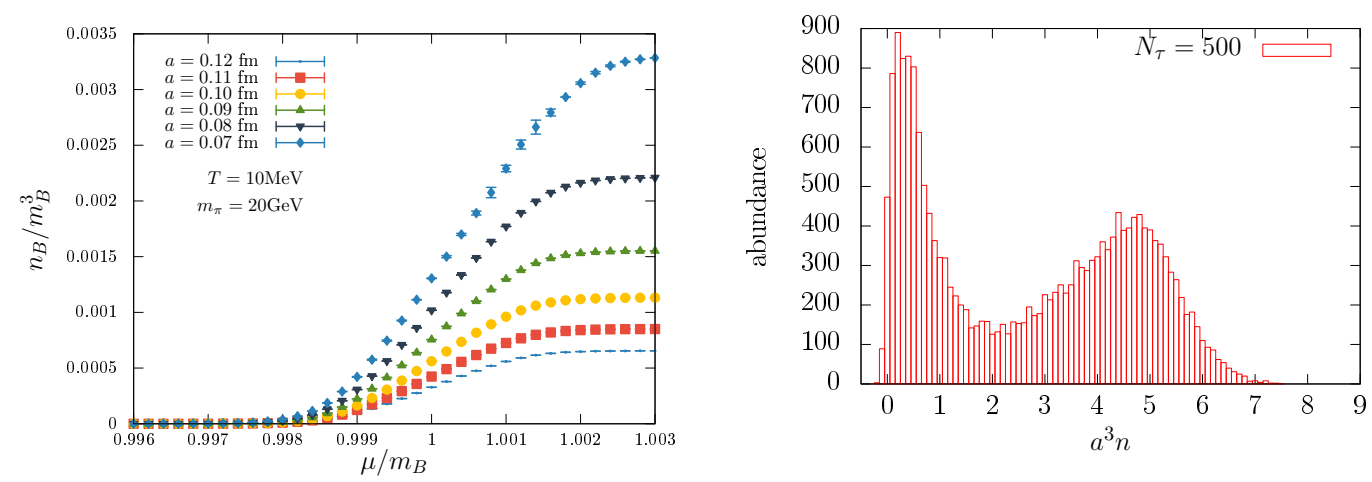

Figure 12: Left: Onset of baryon number (crossover) for heavy quarks and different lattice spacings [72]. Right: First-order onset transition for light quarks [71].

\subsection{Effective theory for heavy quarks}

The representation of QCD in terms of Polyakov loops was developed to characterise the thermal transition in pure gauge theories $[65,66]$ and has been extended to include fermions. Starting point is Wilson's lattice formulation. The effective theory is then derived by a combined expansion in the fundamental character coefficient, which is a known function of the gauge coupling and always smaller than one for finite $\beta$, and the hopping parameter,

$$
u(\beta)=\frac{\beta}{18}+\frac{\beta^{2}}{216}+\ldots<1, \quad \frac{1}{8}<\left(\kappa=\frac{1}{2 a m+8}\right)<\frac{1}{4} .
$$

With the euclidean time extent now absorbed in the remaining temporal Wilson line variables $W(\mathbf{x})$, the result is an effectively $3 d$ theory resembling a continuous spin model [67, 68],

$$
\begin{aligned}
Z= & \int D W \prod_{<\mathbf{x}, \mathbf{y}>}\left[1+\lambda\left(L_{\mathbf{x}} L_{\mathbf{y}}^{*}+L_{\mathbf{x}}^{*} L_{\mathbf{y}}\right)\right] \\
& \times \prod_{\mathbf{x}}\left[1+h_{1} L_{\mathbf{x}}+h_{1}^{2} L_{\mathbf{x}}^{*}+h_{1}^{3}\right]^{2 N_{f}}\left[1+\bar{h}_{1} L_{\mathbf{x}}^{*}+\bar{h}_{1}^{2} L_{\mathbf{x}}+\bar{h}_{1}^{3}\right]^{2 N_{f}} \\
& \times \prod_{<\mathbf{x}, \mathbf{y}>}\left(1-h_{2} \operatorname{Tr} \frac{h_{1} W_{\mathbf{x}}}{1+h_{1} W_{\mathbf{x}}} \operatorname{Tr} \frac{h_{1} W_{\mathbf{y}}}{1+h_{1} W_{\mathbf{y}}}\right)\left(1-h_{2} \operatorname{Tr} \frac{\bar{h}_{1} W_{\mathbf{x}}^{\dagger}}{1+\overline{h_{1} W_{\mathbf{x}}^{\dagger}}} \operatorname{Tr} \frac{\bar{h}_{1} W_{\mathbf{y}}^{\dagger}}{1+\bar{h}_{1} W_{\mathbf{y}}^{\dagger}}\right) \times \ldots
\end{aligned}
$$

The couplings of the effective theory, $\lambda\left(u, \kappa, N_{\tau}\right), h_{1}\left(u, \kappa, \mu, N_{\tau}\right), \overline{h_{1}}(\mu)=h_{1}(-\mu), h_{2}\left(u, \kappa, N_{\tau}\right)$ are (resummed) power series in the original small parameters, and can thus in turn be treated as small expansion parameters for the effective theory. This is illustrated in [69], where the critical coupling for the $S U(3)$ pure gauge theory is extracted from the effective theory by means of series expansion methods. Comparison with the full $4 \mathrm{~d}$ Monte Carlo result shows agreement to better than $10 \%$ for $N_{\tau} \in[2,16]$, for which continuum extrapolations are possible, thus constituting a completely analytic calculation of the deconfinement transition of lattice Yang-Mills theory.

When quarks are included, the heavy mass corner of the Columia plot has been simulated by means of the effective theory. On coarse $N_{\tau}=4$ lattices, the critical line found in full QCD simulations at $\mu=0$ is again accurately reproduced, and the calculation can be extended to finite $\mu$ simulating a flux representation [68]. In this way the deconfinement critical surface is known for any value of $\mu$, Fig. 11 (left), and the phase diagram for heavy quarks looks like Fig. 11 (right). 

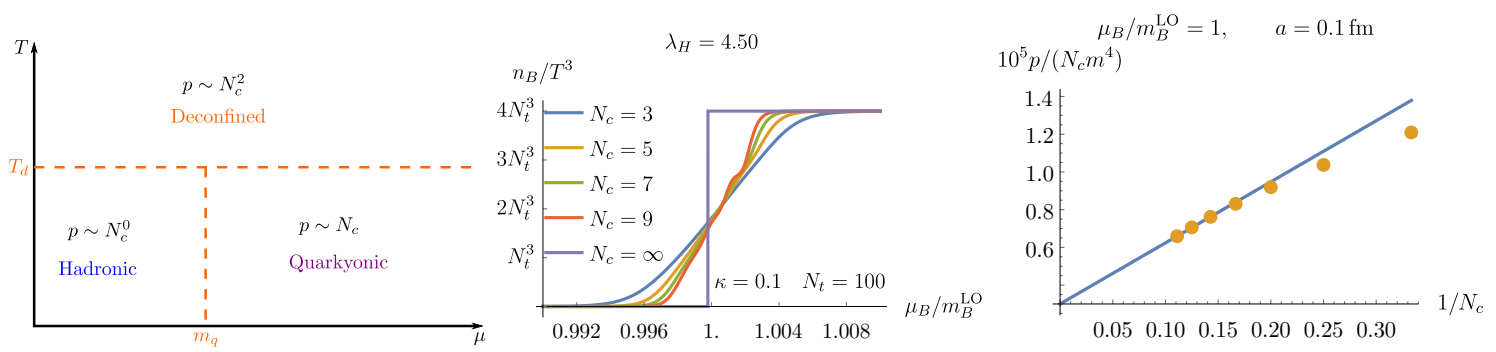

Figure 13: Left: Phase diagram for QCD with $N_{c} \rightarrow \infty$ [73]. Middle: The onset transition becomes first order for large $N_{c}$. Right: $p \sim N_{c}$ for large $N_{c}$ in the baryon condensed region [75].

With the same methods, the cold and dense regime can also be studied and, in particular, the onset transition to condensing baryon matter has been seen explicitly to various orders in the expansions [70, 71, 72]. Fig. 12 (left) shows the baryon density, featuring the "silver blaze property" of staying zero until $\mu_{B} \approx m_{B}$, followed by a sudden rise to lattice saturation, which is the maximal number of quarks per lattice site allowed by the Pauli principle. In continuum units, this lattice artefact moves to infinity by continuum extrapolation, as the figure illustrates. Fig. 12 (left) shows a crossover, whereas for light quarks simulations show a first-order transition Fig. 12 (right) changing to crossover at an endpoint $T_{c}(m)$. Indeed, the binding energy per baryon in the hopping expansion is found to start as $\varepsilon \sim \kappa^{2}$ [71], i.e. it decreases with growing quark mass to zero in the static limit, as one also expects from Yukawa potentials in nuclear physics. Hence, the end point of the nuclear liquid gas transition, $T_{c}(m)$, decreases with mass.

\subsection{Large $N_{c}$}

In yet another corner of QCD parameter space, interesting conjectures concerning the QCD phase structure were based on large $N_{c}$ arguments [73]. In particular, the phase diagram in the large $N_{c}$ limit was argued to look as in Fig. 13 (left). With fermion contributions suppressed, the deconfinement transition is a straight line separating the plasma phase, where the pressure scales as $p \sim N_{c}^{2}$, from the hadron gas phase, where it scales as $p \sim N_{c}^{0}$. In [73] it is argued that at finite density there should then be a third phase with $p \sim N_{c}$, which was termed quarkyonic since it shows aspects of both baryon and quark matter. In particular, the fermi sea at low temperatures is argued to be composed of a baryonic shell of thickness $\sim \Lambda_{Q C D}$, and quark matter inside.

The effective theory of the previous section can be derived for a general number of colours [74]. For large $N_{c}$, the baryon mass is $m_{B} \sim N_{c}$, so the constituent quark mass should not matter and the cold and dense region for large $N_{c}$ is accessible to direct calculation [75]. It was found that the baryon onset transition steepens with $N_{c}$, to become first-order in the large $N_{c}$ limit, Fig. 13 (middle). Furthermore, through three orders in the hopping expansion, the pressure scales as $p \sim N_{c}$, suggesting this to be a property to all orders. This scaling is reproduced with a leading correction even for $N_{c}=3-9$, right after the onset transition Fig. 13 (right). The large $N_{c}$ phase diagram Fig. 13 (left) is thus continuously obtained from Fig. 11 (right) by increasing $N_{c}$. Note also, that a lattice filling with baryon number smoothly changes from baryon matter (at the onset of condensation) to quark matter (at saturation) as a function of $\mu_{B}$, which is consistent with the picture of quarkyonic matter. For light quarks, there may be in addition a chiral transition. 

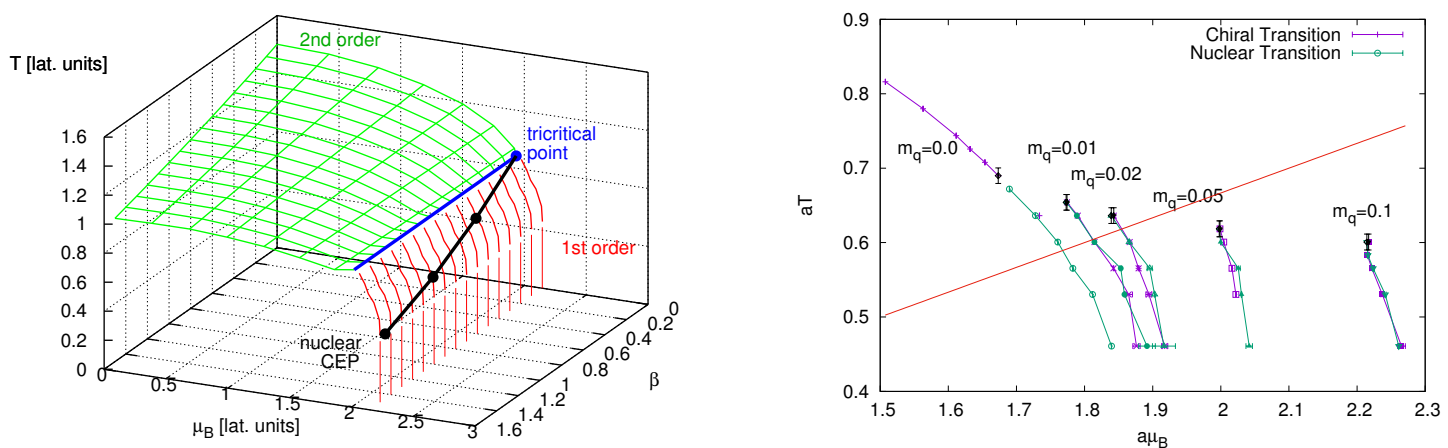

Figure 14: Left: Chiral phase transition and onset transition to baryon condensation for $m=0$ [82]. Right: Mass dependence of the chiral and baryon onset transition in the strong coupling limit [83].

\subsection{Effective theory for light quarks}

In this case the starting point is the lattice action with staggered fermions. After a strong coupling expansion in powers of $\beta$, the gauge integration is done to leave a fermionic theory,

$$
\begin{aligned}
Z & =\int D \bar{\psi} D \psi D U e^{-S_{g}[U]-S_{f}[U, \bar{\psi}, \psi]}=\int D \bar{\psi} D \psi Z_{f}\left\langle e^{-S_{g}[U]}\right\rangle_{Z_{f}}, \\
Z_{f} & =\int D U e^{-S_{f}[U, \bar{\psi}, \psi]}, \quad\left\langle e^{-S_{g}[U]}\right\rangle_{Z_{f}}=1+\left\langle S_{g}[U]\right\rangle_{Z_{f}}+O\left(\beta^{2}\right) .
\end{aligned}
$$

Early mean field [76] and Monte Carlo [77] studies based on a polymer representation have been restricted to the strong coupling limit, $\beta=0$. More recent simulations are done with a worm algorithm [79]. Note that anisotropic lattices are necessary for $\beta=0$ in order to tune temperature.

After integrating over the fermions also, one arrives at a dual formulation in terms of monomers, dimers, world lines and world sheets, which for isotropic lattices reads [80, 81],

$$
Z\left(m_{q}, \mu\right)=\sum_{\left\{k, n, l, n_{p}\right\}} \underbrace{\prod_{b=(x, v)} \frac{\left(N_{c}-k_{b}\right) !}{N_{c} !\left(k_{b}-\left|f_{b}\right|\right) !}}_{\text {meson hoppings }} \underbrace{\prod_{x} \frac{N_{c} !}{n_{x} !}\left(2 a m_{q}\right)^{n_{x}}}_{\text {chiral condensate }} \underbrace{\prod_{l_{3}} w\left(l_{3}, \mu\right) \prod_{l_{f}} \tilde{w}\left(l_{f}, \mu\right)}_{\text {baryon hoppings }} \underbrace{\prod_{p} \frac{\left(\frac{\beta}{2 N_{c}}\right)^{n_{p}+\bar{n}_{p}}}{n_{p} ! \bar{n}_{p} !}}_{\text {gluon propagation }} .
$$

Note that this formulation in general also contains negative weights, but the resulting sign problem is mild enough to be handled by reweighting techniques. A particular advantage of this formulation is the feasibility to simulate the chiral limit as well as finite mass, a drawback is that gauge corrections are more difficult to include.

Fig. 14 (left) shows the phase diagram for the chiral limit, both for $\beta=0$ and with leading linear gauge corrections included [82]. As expected, there always is a non-analytic chiral phase transition, with a tricritical point where the first-order transition at finite density meets the secondorder line. In the strong coupling limit, this tricritical point coincides with the end point of the nuclear liquid gas transition. When gauge corrections are switched on, these start splitting up, but surprisingly the first-order lines of the chiral and nuclear transitions are still indistinguishably close. Fig. 14 (right) shows the strong coupling limit, but now with finite quark mass switched on. The second-order transition line changes to crossover, as expected. Note the decreasing $T_{c}(m)$ of the end point, which is in qualitative agreement with the finding for heavy quarks. The end point 

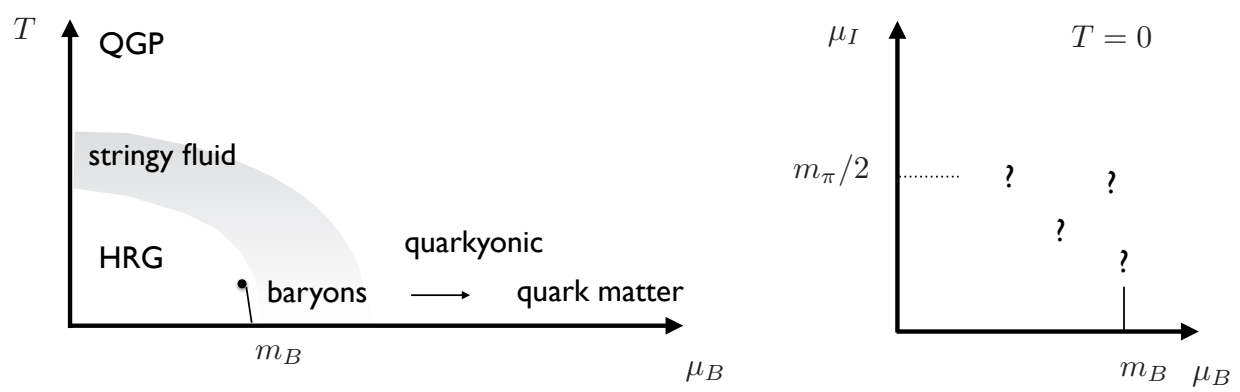

Figure 15: Phase diagram with only those qualitative features, which are seen in lattice simulations so far.

quickly moves to $\mu_{B} \gtrsim 3 T$, which is again consistent with all previously reported results. First simulations including both, gauge corrections as well as finite mass, are also available [84]. Note also that, in the continuum, this effective theory represents $N_{f}=4$ QCD if no rooting is applied.

\section{Conclusions}

Because direct simulations are impossible, determining the nature of the QCD transition in the chiral limit as well as at finite baryon density remains extraordinarily difficult. Nevertheless, systematic studies of QCD transitions in accessible regions of parameter space are beginning to constrain the possible phase diagram. In particular, the strength of the chiral transition weakens with decreasing $N_{f}$ and with decreasing lattice spacing. For the chiral limit with $N_{f}=2$ and surprisingly also for $N_{f}=3$, this implies either a second-order transition, or a first-order transition disappearing at excessively small quark masses. The chiral transition also weakens, at least initially, when a real baryon chemical potential is switched on. For physical quark masses, there is no conclusive sign of criticality from the lattice for $\mu_{B} \lesssim 3 T$. This is consistent with recent DysonSchwinger [85] and functional renormalisation group [86] results, which predict a critical point in the range $\mu_{B} / T \approx 4-6$, with however still uncertain systematics in that chemical potential range.

The cold and dense regime $\mu_{B} / T \gg 1$, progress is being made with analytically derived effective lattice theories, which represent QCD in complementary parameter regions with either heavy quarks or at strong coupling. They unambiguously show the silver blaze behaviour at $T=0$, followed by a first-order transition to baryon condensation with a critical end point, which for physical parameter values will represent the nuclear liquid gas transition. At finite isospin chemical potential and low temperatures, a second-order transition to a pion condensed phase is seen for physical quark masses and in the continuum. These features are indicated in the phase diagram Fig. 15.

Other developments concern the physical degrees of freedom near the thermal transition. An emergent chiral spin symmetry in the temperature range from the crossover to $\sim 1 \mathrm{GeV}$ suggests light quarks still bound by colour-electric strings, and the symmetry gets amplified by baryon chemical potential. At low temperatures, there is the onset transition to baryon matter, which smoothly turns into a quarkyonic regime (defined by $p \sim N_{c}$ ) that, at least in principle, allows a continuous interpolation from baryon matter to quark matter. The band in the phase diagram Fig. 15 thus indicates a region, where the dynamics changes very gradually and the degrees of freedom still resemble the hadronic ones. In conclusion, the lattice is beginning to see some structure in the QCD phase diagram. Intriguingly, a non-analytic chiral phase transition is neither required nor ruled out by lattice data at this stage, and remains an exciting subject of research. 


\section{Acknowledgments}

The author is grateful to B. Brandt for helpful discussions and acknowledges support by the Deutsche Forschungsgemeinschaft (DFG) through the grant CRC-TR 211 "Strong-interaction matter under extreme conditions", as well as by the Helmholtz International Center for FAIR within the LOEWE program of the State of Hesse.

\section{References}

[1] P. de Forcrand, PoS LAT 2009 (2009) 010 [arXiv:1005.0539 [hep-lat]].

[2] G. Boyd et al., Nucl. Phys. B 469 (1996) 419 [hep-lat/9602007].

[3] R. D. Pisarski and F. Wilczek, Phys. Rev. D 29 (1984) 338.

[4] A. Pelissetto and E. Vicari, Phys. Rev. D 88 (2013) no.10, 105018 [arXiv:1309.5446 [hep-lat]].

[5] F. Karsch, E. Laermann and C. Schmidt, Phys. Lett. B 520 (2001) 41 [hep-lat/0107020].

[6] P. de Forcrand and O. Philipsen, Nucl. Phys. B 673 (2003) 170 [hep-lat/0307020].

[7] X. Y. Jin et al., Phys. Rev. D 91 (2015) no.1, 014508 [arXiv:1411.7461 [hep-lat]].

[8] C. Bonati et al., Phys. Rev. D 90 (2014) no.7, 074030 [arXiv:1408.5086 [hep-lat]].

[9] F. Cuteri, O. Philipsen and A. Sciarra, Phys. Rev. D 97 (2018) no.11, 114511 [arXiv:1711.05658 [hep-lat]].

[10] O. Philipsen and C. Pinke, Phys. Rev. D 93 (2016) no.11, 114507 [arXiv:1602.06129 [hep-lat]].

[11] A. Bazavov et al., Phys. Rev. D 95 (2017) no.7, 074505 [arXiv:1701.03548 [hep-lat]].

[12] Y. Aoki et al., Nature 443 (2006) 675 [hep-lat/0611014].

[13] F. Cuteri, O. Philipsen and A. Sciarra, PoS LATTICE 2018 (2018) 170 [arXiv:1811.03840 [hep-lat]].

[14] P. de Forcrand and M. D'Elia, PoS LATTICE 2016 (2017) 081 [arXiv:1702.00330 [hep-lat]].

[15] X. Y. Jin et al., Phys. Rev. D 96 (2017) no.3, 034523 [arXiv:1706.01178 [hep-lat]].

[16] H. T. Ding et al., PoS LATTICE 2018 (2019) 171 [arXiv:1905.11610 [hep-lat]].

[17] H. T. Ding et al., Phys. Rev. Lett. 123 (2019) no.6, 062002 [arXiv:1903.04801 [hep-lat]].

[18] G. Endrödi and L. Gonglach, PoS LATTICE 2018 (2018) 172 [arXiv:1810.09173 [hep-lat]].

[19] S. Chandrasekharan and A. C. Mehta, Phys. Rev. Lett. 99 (2007) 142004 [arXiv:0705.0617 [hep-lat]].

[20] M. I. Buchoff et al., Phys. Rev. D 89 (2014) no.5, 054514 [arXiv:1309.4149 [hep-lat]].

[21] V. Dick et al., Phys. Rev. D 91 (2015) no.9, 094504 [arXiv:1502.06190 [hep-lat]].

[22] B. B. Brandt et al., JHEP 1612 (2016) 158 [arXiv:1608.06882 [hep-lat]].

[23] A. Bazavov et al., Phys. Rev. D 100 (2019) no.9, 094510 [arXiv:1908.09552 [hep-lat]].

[24] K. Suzuki et al., EPJ Web Conf. 175 (2018) 07025 [arXiv:1711.09239 [hep-lat]].

[25] A. G. Nicola, J. Ruiz De Elvira, A. Vioque-Rodríguez and S. Ferreres-Solé, PoS Confinement 2018 (2019) 153 [arXiv:1812.04516 [hep-ph]].

[26] P. de Forcrand and O. Philipsen, Nucl. Phys. B 642 (2002) 290 [hep-lat/0205016]. 
[27] M. D’Elia and M. P. Lombardo, Phys. Rev. D 67 (2003) 014505 [hep-lat/0209146].

[28] A. Roberge and N. Weiss, Nucl. Phys. B 275 (1986) 734.

[29] P. de Forcrand and O. Philipsen, Phys. Rev. Lett. 105 (2010) 152001 [arXiv:1004.3144 [hep-lat]].

[30] C. Bonati, G. Cossu, M. D’Elia and F. Sanfilippo, Phys. Rev. D 83 (2011) 054505 [arXiv:1011.4515 [hep-lat]].

[31] O. Philipsen and C. Pinke, Phys. Rev. D 89 (2014) no.9, 094504 [arXiv:1402.0838 [hep-lat]].

[32] P. de Forcrand and O. Philipsen, JHEP 0701 (2007) 077 [hep-lat/0607017].

[33] O. Philipsen and A. Sciarra, arXiv:1909.12253 [hep-lat].

[34] C. Czaban et al., Phys. Rev. D 93 (2016) no.5, 054507 [arXiv:1512.07180 [hep-lat]].

[35] C. Bonati et al., Phys. Rev. D 99 (2019) no.1, 014502 [arXiv:1807.02106 [hep-lat]].

[36] J. Goswami et al., PoS CORFU 2018 (2019) 162 [arXiv:1905.03625 [hep-lat]].

[37] L. Y. Glozman, Eur. Phys. J. A 51 (2015) no.3, 27 [arXiv:1407.2798 [hep-ph]].

[38] C. Rohrhofer et al., Phys. Rev. D 100 (2019) no.1, 014502 [arXiv:1902.03191 [hep-lat]].

[39] C. Rohrhofer, Y. Aoki, L. Y. Glozman and S. Hashimoto, arXiv:1909.00927 [hep-lat].

[40] P. B. Arnold and L. G. Yaffe, Phys. Rev. D 52 (1995) 7208 [hep-ph/9508280].

[41] A. Hart, M. Laine and O. Philipsen, Nucl. Phys. B 586 (2000) 443 [hep-ph/0004060].

[42] Z. Fodor and S. D. Katz, Phys. Lett. B 534 (2002) 87 [hep-lat/0104001].

[43] C. R. Allton et al., Phys. Rev. D 66 (2002) 074507 [hep-lat/0204010].

[44] V. Vovchenko, J. Steinheimer, O. Philipsen and H. Stoecker, PoS CORFU 2018 (2019) 199 [arXiv:1905.01031 [hep-ph]].

[45] Z. Fodor et al., Nucl. Phys. A 982 (2019) 843.

[46] C. Ratti, PoS LATTICE 2018 (2019) 004.

[47] D. Sexty, Phys. Rev. D 100 (2019) no.7, 074503 [arXiv:1907.08712 [hep-lat]].

[48] C. Bonati et al., Nucl. Phys. A 982 (2019) 198 [arXiv:1807.10026 [hep-lat]].

[49] C. Bonati et al., Phys. Rev. D 98 (2018) no.5, 054510 [arXiv:1805.02960 [hep-lat]].

[50] A. Bazavov et al., Phys. Lett. B 795 (2019) 15 [arXiv:1812.08235 [hep-lat]].

[51] A. Bazavov et al., Phys. Rev. D 95 (2017) no.5, 054504 [arXiv:1701.04325 [hep-lat]].

[52] V. Vovchenko, J. Steinheimer, O. Philipsen and H. Stoecker, Phys. Rev. D 97 (2018) no.11, 114030 [arXiv:1711.01261 [hep-ph]].

[53] V. Vovchenko et al., Phys. Lett. B 775 (2017) 71 [arXiv:1708.02852 [hep-ph]].

[54] G. A. Almási, B. Friman, K. Morita and K. Redlich, Phys. Lett. B 793 (2019) 19 [arXiv:1902.05457 [hep-ph]].

[55] G. N. Mercer and A. J. Roberts, SIAM J. Appl. Math. 50, 1547 (1990).

[56] M. Giordano and A. Pásztor, Phys. Rev. D 99 (2019) no.11, 114510 [arXiv:1904.01974 [hep-lat]].

[57] Z. Fodor and S. D. Katz, JHEP 0203 (2002) 014 [hep-lat/0106002]. 
[58] M. Giordano et al., arXiv:1911.00043 [hep-lat].

[59] M. G. Alford, A. Kapustin and F. Wilczek, Phys. Rev. D 59 (1999) 054502 [hep-lat/9807039].

[60] J. B. Kogut and D. K. Sinclair, Phys. Rev. D 66 (2002) 034505 [hep-lat/0202028].

[61] P. de Forcrand, M. A. Stephanov and U. Wenger, PoS LATTICE 2007 (2007) 237 [arXiv:0711.0023 [hep-lat]].

[62] P. Cea et al., PoS LATTICE 2012 (2012) 067 [arXiv:1210.5896 [hep-lat]].

[63] B. B. Brandt, G. Endrodi and S. Schmalzbauer, Phys. Rev. D 97 (2018) no.5, 054514 [arXiv:1712.08190 [hep-lat]].

[64] S. Schmalzbauer et al., these proceedings, arXiv:1911.12197 [hep-lat].

[65] J. Polonyi and K. Szlachanyi, Phys. Lett. 110B (1982) 395.

[66] B. Svetitsky and L. G. Yaffe, Nucl. Phys. B 210 (1982) 423.

[67] J. Langelage, S. Lottini and O. Philipsen, JHEP 1102 (2011) 057 Erratum: [JHEP 1107 (2011) 014] [arXiv:1010.0951 [hep-lat]].

[68] M. Fromm, J. Langelage, S. Lottini and O. Philipsen, JHEP 1201 (2012) 042 [arXiv:1111.4953 [hep-lat]].

[69] J. Kim, A. Q. Pham, O. Philipsen and J. Scheunert, arXiv:1912.01705 [hep-lat].

[70] M. Fromm et al., Phys. Rev. Lett. 110 (2013) no.12, 122001 [arXiv:1207.3005 [hep-lat]].

[71] J. Langelage, M. Neuman and O. Philipsen, JHEP 1409 (2014) 131 [arXiv:1403.4162 [hep-lat]].

[72] J. Glesaaen, M. Neuman and O. Philipsen, JHEP 1603 (2016) 100 [arXiv:1512.05195 [hep-lat]].

[73] L. McLerran and R. D. Pisarski, Nucl. Phys. A 796 (2007) 83 [arXiv:0706.2191 [hep-ph]].

[74] A. S. Christensen, J. C. Myers and P. D. Pedersen, JHEP 1402 (2014) 028 [arXiv:1312.3519 [hep-lat]].

[75] O. Philipsen and J. Scheunert, JHEP 1911 (2019) 022 [arXiv:1908.03136 [hep-lat]].

[76] N. Kawamoto and J. Smit, Nucl. Phys. B 192 (1981) 100.

[77] F. Karsch and K. H. Mutter, Nucl. Phys. B 313 (1989) 541.

[78] P. de Forcrand, W. Unger and H. Vairinhos, Phys. Rev. D 97 (2018) no.3, 034512 [arXiv:1710.00611 [hep-lat]].

[79] P. de Forcrand and M. Fromm, Phys. Rev. Lett. 104 (2010) 112005 [arXiv:0907.1915 [hep-lat]].

[80] G. Gagliardi, J. Kim and W. Unger, EPJ Web Conf. 175 (2018) 07047 [arXiv:1710.07564 [hep-lat]].

[81] G. Gagliardi and W. Unger, arXiv:1911.08389 [hep-lat].

[82] P. de Forcrand, J. Langelage, O. Philipsen and W. Unger, Phys. Rev. Lett. 113 (2014) no.15, 152002 [arXiv:1406.4397 [hep-lat]].

[83] J. Kim and W. Unger, PoS LATTICE 2016 (2016) 035 [arXiv:1611.09120 [hep-lat]].

[84] J. Kim, O. Philipsen and W. Unger, arXiv:1912.00822 [hep-lat].

[85] C. S. Fischer, Prog. Part. Nucl. Phys. 105 (2019) 1 [arXiv:1810.12938 [hep-ph]].

[86] W. j. Fu, J. M. Pawlowski and F. Rennecke, arXiv:1909.02991 [hep-ph]. 